\title{
Phase study of oscillatory resistances in microwave-irradiated-and dark-GaAs/AlGaAs devices: Indications of an unfamiliar class of the integral quantum Hall effect
}

\section{Citation}

Mani, R. G., W. B. Johnson, V. Umansky, V. Narayanamurti, and K. Ploog. 2009. "Phase Study of Oscillatory Resistances in Microwave-Irradiated-and Dark-GaAs/AlGaAs Devices: Indications of an Unfamiliar Class of the Integral Quantum Hall Effect." Physical Review B 79 (20). https:// doi.org/10.1103/physrevb.79.205320.

\section{Permanent link}

http://nrs.harvard.edu/urn-3:HUL.InstRepos:41417253

\section{Terms of Use}

This article was downloaded from Harvard University's DASH repository, and is made available under the terms and conditions applicable to Other Posted Material, as set forth at http:// nrs.harvard.edu/urn-3:HUL.InstRepos:dash.current.terms-of-use\#LAA

\section{Share Your Story}

The Harvard community has made this article openly available.

Please share how this access benefits you. Submit a story.

\section{Accessibility}




\title{
Phase study of oscillatory resistances in microwave-irradiated- and dark-GaAs/AIGaAs devices: Indications of an unfamiliar class of the integral quantum Hall effect
}

\author{
R. G. Mani, ${ }^{1}$ W. B. Johnson, ${ }^{2}$ V. Umansky, ${ }^{3}$ V. Narayanamurti, ${ }^{4}$ and K. Ploog ${ }^{5}$ \\ ${ }^{1}$ Department of Physics and Astronomy, Georgia State University, 29 Peachtree Center Avenue, Atlanta, Georgia 30303, USA \\ ${ }^{2}$ Laboratory for Physical Sciences, University of Maryland, College Park, Maryland 20740, USA \\ ${ }^{3}$ Braun Center for Submicron Research, Weizmann Institute, Rehovot 76100, Israel \\ ${ }^{4}$ SEAS, Harvard University, 29 Oxford Street, Cambridge, Massachusetts 02138, USA \\ ${ }^{5}$ Paul-Drude-Institut für Festkörperelektronik, Hausvogteiplatz, 5-7, 10117 Berlin, Germany
}

(Received 25 February 2009; published 27 May 2009)

\begin{abstract}
We report the experimental results from a dark study and a photoexcited study of the high-mobility GaAs/ AlGaAs system at large filling factors, $\nu$. At large $\nu$, the dark study indicates several distinct phase relations ("type 1," "type 2," and "type 3") between the oscillatory diagonal and Hall resistances, as the canonical integral quantum Hall effect (IQHE) is manifested in the type 1 case of approximately orthogonal diagonal and Hall resistance oscillations. Surprisingly, the investigation indicates quantum Hall plateaus also in the type 3 case characterized by approximately "antiphase" Hall and diagonal resistance oscillations, suggesting an unfamiliar and distinct class of IQHE. Transport studies under microwave photoexcitation exhibit radiationinduced magnetoresistance oscillations in both the diagonal, $R_{x x}$, and off-diagonal, $R_{x y}$, resistances. Further, when the radiation-induced magnetoresistance oscillations extend into the quantum Hall regime, there occurs a radiation-induced nonmonotonic variation in the amplitude of Shubnikov-de Haas $(\mathrm{SdH})$ oscillations in $R_{x x}$ vs $B$, and a nonmonotonic variation in the width of the quantum Hall plateaus in $R_{x y}$. The latter effect leads into the vanishing of IQHE at the minima of the radiation-induced $R_{x x}$ oscillations with increased photoexcitation. We reason that the mechanism which is responsible for producing the nonmonotonic variation in the amplitude of $\mathrm{SdH}$ oscillations in $R_{x x}$ under photoexcitation is also responsible for eliminating, under photoexcitation, the type 3 associated IQHE in the high-mobility specimen.
\end{abstract}

DOI: $10.1103 /$ PhysRevB.79.205320

PACS number(s): 73.43.Qt, 73.21.-b, 73.40.-c

\section{INTRODUCTION}

A two-dimensional electron system (2DES) at high magnetic fields, $B$, and low temperatures, $T$, exhibits the integral quantum Hall effect (IQHE), which is characterized by plateaus in the Hall resistance $R_{x y}$ vs $B$, at $R_{x y}=h / i e^{2}$, with $i$ $=1,2,3, \ldots$ and concurrent vanishing diagonal resistance $R_{x x}$ as $T \rightarrow 0 \mathrm{~K}$, in the vicinity of integral filling factors of Landau levels, i.e., $\nu \approx i .^{1-3}$ With the increase in the electron mobility, $\mu$, at a given electron density, $n$, and low $T$, IQHE plateaus typically become narrower as fractional quantum Hall effects (FQHE) appear in the vicinity of $\nu \approx p / q$, at $R_{x y}=h /\left[(p / q) e^{2}\right]$, where $p / q$ denotes mostly odddenominator rational fractions. ${ }^{2,3}$ Experimental studies of the highest mobility specimens have typically focused upon FQHE and other novel phases. ${ }^{2-5}$ Meanwhile, the possibility of unfamiliar and distinct variations of IQHE that might appear with the canonical effect in the reduced-disorder specimen, especially at large $\nu$, has been largely unanticipated. In the first dark-study part of this paper, we show that three distinct phase relationships can occur between the oscillatory diagonal and Hall resistances in the high-mobility dark specimen at $\nu>5$, and that IQHE can be manifested in two of these variations. The results therefore suggest another distinct class of IQHE, as they provide insight into the origin of oscillatory variations in the Hall effect, and their evolution into Hall plateaus, in the low- $B$ large- $\nu$ regime of the radiation-induced zero-resistance states in the photoexcited high-mobility 2 DES. ${ }^{6-49}$

Transport studies of photoexcited two-dimensional electron systems have become a topic of interest following the discovery of novel radiation-induced zero-resistance states in the GaAs/AlGaAs system at high filling factors. ${ }^{6,16}$ The characteristic field $B_{f}$ for these zero-resistance states and associated magnetoresistance oscillations is a linear function of the radiation frequency, $f$, i.e., $B_{f}=2 \pi f m^{*} / e$, and, therefore, an increase in $f$ could be expected to bring about an overlap of the radiation-induced zero-resistance states and the quantum Hall effect. In this case, a topic of interest is the interplay between the radiation-induced phenomena and quantum Hall effect. Hence, in the second part of the paper, we follow up the above-mentioned dark study of oscillatory resistances and quantum Hall effect at large filling factors, by examining also the influence of microwave photoexcitation. Here, the experimental results show that vanishing resistance induced by photoexcitation helps to replace the quantum Hall effect by an ordinary Hall effect over broad magnetic field intervals in the vicinity of the radiation-induced oscillatory $R_{x x}$ minima. The results also identify a strong correlation between the vanishing of Shubnikov-de Haas (SdH) oscillations in $R_{x x}$ and the narrowing of Hall plateaus in $R_{x y}$ under photoexcitation. Comparative plots of the oscillatory diagonal and off-diagonal resistances that help to establish this correlation also serve to confirm the observations made on the dark specimen, namely, that IQHE sometimes goes together with "type 3" resistance oscillations in the highmobility specimen at large $\nu$.

\section{EXPERIMENT}

Simultaneous low-frequency ac lock-in based electrical measurements of $R_{x x}$ and $R_{x y}$ were carried out on GaAs/ 
AlGaAs single heterojunctions at $T>0.45 \mathrm{~K}$, with matched lock-in time constants, and sufficiently slow $B$-field sweep rates. The $B$ field was calibrated by ESR of DPPH. ${ }^{12,29}$

Shubnikov-de Haas oscillations and associated IQHE became weaker, as usual, at higher $T$, and few oscillations or Hall plateaus were evident for $\nu>20$ at $T>1.7 \mathrm{~K}$ in the dark study. Thus, we focused upon $0.45<T<1.7 \mathrm{~K}$, where $T$-induced changes in the phase relations were not discerned and, further, beats were not observed in the $\mathrm{SdH}$ oscillations. The observed phase differences were verified not to be experimental artifacts originating from the choice of experimental parameters such as the $B$-sweep rate, the data acquisition rate, lock-in integration time, and other typical variables. The observed phase relations also did not show an obvious dependence on the sample geometry, or type (Au-Ge/Ni or In) of contacts. The reported phase relation between the oscillatory Hall and diagonal resistances could often be identified by eye. Yet, we have utilized background subtraction here for the sake of presentation, mainly to realize overlays in the figures, for phase comparison. An explicit example of the background subtraction procedure is illustrated in the Appendix. Finally, although the mobility $\mu$ has been provided, $\mu$ alone seems not to be sufficient for classifying the observed phenomena in high- $\mu$ specimens. Here, the high-mobility condition was realized by brief illumination with a red LED.

The second part of the study, i.e., the photoexcited study, ${ }^{6}$ followed the same methods as the dark study with the difference that microwaves introduced via a rectangular waveguide served to irradiate the specimen. Here, the radiation intensity was adjusted externally as desired.

\section{PART 1: THE DARK STUDY}

\section{A. Background: "type 1" phase relation in the canonical IQHE at large $\nu$}

To review the basics, Fig. 1(a) exhibits measurements from a typical low-mobility Hall bar specimen with $n=3.2$ $\times 10^{11} \mathrm{~cm}^{-2}$ and $\mu=0.4 \times 10^{6} \mathrm{~cm}^{2} / \mathrm{V} \mathrm{s}$. Here, as is usual with IQHE, large amplitude SdH oscillations in $R_{x x}$ lead into zero-resistance states with increasing $B$, as $R_{x y}$ exhibits plateaus at $R_{x y}=h / i e^{2}$ for $\nu \approx i$, with $i=2,4,6, \ldots$ This canonical low-mobility IQHE system is known to follow a resistivity/ resistance rule, ${ }^{50,51}$ at a each $T,{ }^{52}$ whereby $R_{x x} \propto B\left[d R_{x y} / d B\right]$ and $d R_{x y} / d B$ is the $B$-field derivative of $R_{x y} .{ }^{50}$ In order to check the validity of this rule, Fig. $1(\mathrm{~b})$ exhibits $B\left[d R_{x y} / d B\right]$, which is then to be compared with the $R_{x x}$ shown in Fig. 1(a). Such a comparison suggests general consistency between the observed results and the suggested rule..$^{50-52}$

For the sake of further analysis, Fig. 1(c) shows the oscillatory part of the diagonal $\left(\Delta R_{x x}\right)$ and the off-diagonal Hall $\left(\Delta R_{x y}\right)$ resistances vs $\nu$. Here, $\Delta R_{x y}=R_{x y}-R_{x y}^{\text {back }}$ and $\Delta R_{x x}$ $=R_{x x}-R_{x x}^{\mathrm{back}}$, as $R_{x y}^{\mathrm{back}}$ and $R_{x x}^{\mathrm{back}}$ are the background resistances shown in Fig. 1(a). Note that, sometimes, we shall also refer to this oscillatory $\Delta R_{x y}$ as " $\mathrm{SdH}$ oscillations," for lack of a better term, simply to avoid confusing it with the radiationinduced oscillations in $R_{x y}$. The reader should bear in mind that these $\mathrm{SdH}$ oscillations in $R_{x y}$ are sometimes just another manifestation of IQHE. As evident from Fig. 1(c), the quan-
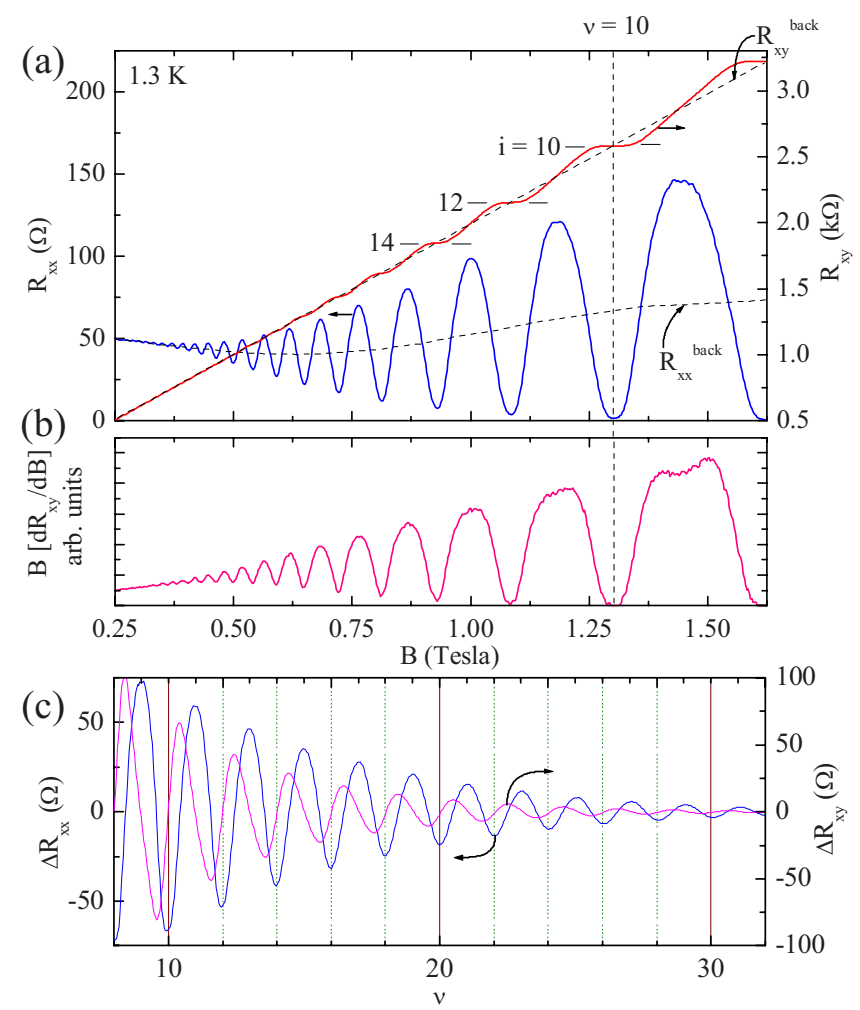

FIG. 1. (Color online) Canonical IQHE and type 1 oscillations: (a) Hall plateaus at $R_{x y}=h / i e^{2}$, i.e., IQHE's, coincide with minima in the diagonal resistance $R_{x x}$ in a GaAs/AlGaAs Hall bar device. (b) A comparison of $B\left[d R_{x y} / d B\right]$ shown here in Fig. 1(b) with $R_{x x}$ in Fig. 1(a) suggests that $R_{x x} \propto B[d R x y / d B]$, as per the resistivity rule (Ref. 50). (c) A canonical quantum Hall system at large $\nu$ also exhibits an approximately $\pi / 2$ phase shift between the oscillatory parts of $R_{x x}$ and $R_{x y}$.

tum Hall characteristics of Fig. 1(a) yield approximately orthogonal oscillations in $\Delta R_{x x}$ and $\Delta R_{x y}$ such that $\Delta R_{x x} \approx$ $-\cos (2 \pi[\nu / 2])$ and $\Delta R_{x y} \approx \sin (2 \pi[\nu / 2])$. This phase description becomes more appropriate at higher filling factors as the harmonic content in the oscillations is reduced and the oscillations attain the appearance of exponentially damped sine/ cosine waves. We denote the quantum Hall features of Figs. $1(\mathrm{a})-1(\mathrm{c})$ as type 1 characteristics and present the essentials in Fig. 2(a).

\section{B. Summary of the other observable phase relations at large $\nu$}

This study reports on other observable phase relations in the high-mobility 2DES. We find, for instance, a "type 2" phase relation, where $\left|R_{x y}\right|$ is enhanced (suppressed) at the $R_{x x}$ oscillation peaks (valleys) and the $\Delta R_{x y}$ oscillations are in phase with the $R_{x x}$ or $\Delta R_{x x}$ oscillations, as shown in Fig. 2(b). There also occurs the more remarkable "type 3" case where $\left|R_{x y}\right|$ is enhanced (suppressed) at the $R_{x x} \mathrm{SdH}$ oscillation minima (maxima) and the $\Delta R_{x y}$ oscillations are phaseshifted by approximately " $\pi$ " with respect to the $R_{x x}$ or $\Delta R_{x x}$ oscillations, as shown in Fig. 2(c). Both type 2 and type 3 oscillations show variance from the resistivity/resistance rule for quantum Hall systems. ${ }^{50}$ Here, we survey these experimentally observed phase relations and related crossovers in 
(a)

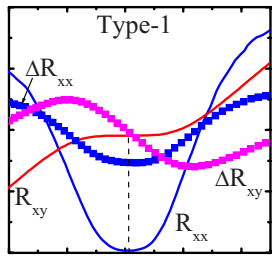

B (arb. units)

(b)

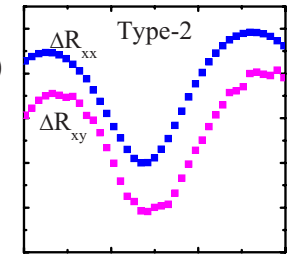

B (arb. units)

(c)

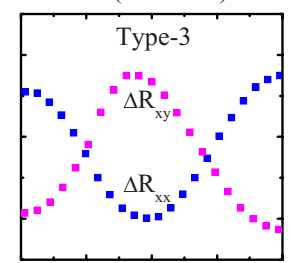

B (arb. units)

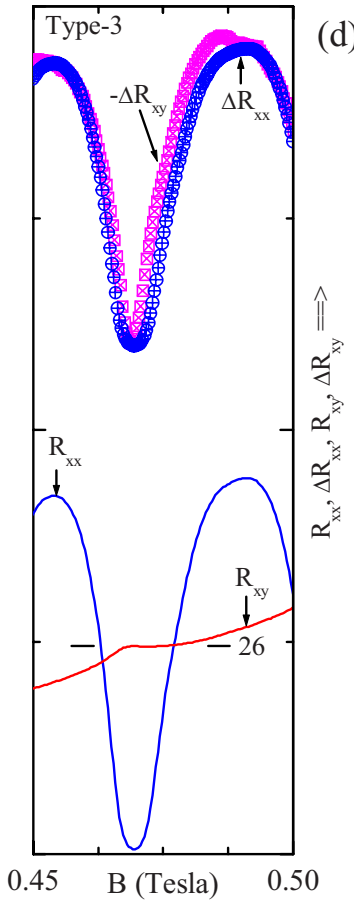

FIG. 2. (Color online) In this figure, panels (a)-(c) exhibit, using representative data, the three types of magnetoresistance oscillations that are examined in this study while panel (d) illustrates an experimental observation of IQHE observed in the case of type 3 oscillations. (a) This panel shows the approximate-orthogonality observed between the $\Delta R_{x x}$ and $\Delta R_{x y}$ oscillations at large filling factors in the usual IQHE, see Fig. 1(c). (b) This panel illustrates the "in-phase" (type 2) oscillations in $\Delta R_{x x}$ and $\Delta R_{x y}$ at large filling factors, which have been observed in this study. (c) This panel presents the approximately $\pi$ phase shift between $\Delta R_{x x}$ and $\Delta R_{x y}$ oscillations that characterizes type 3 oscillations, also observed at large $\nu$ in this study. (d) At the top, this panel exhibits type 3 oscillations at large filling factors. The bottom part of panel (d) illustrates the associated IQHE.

the high-mobility 2DES, and then focus on the type 3 case, which also brings with it, remarkably, a distinct and unfamiliar class of IQHE that is illustrated in Fig. 2(d).

\section{Results}

\section{Type 1-type 2 crossover and type 2 phase relation at large $\nu$}

For a high-mobility specimen with $n=3 \times 10^{11} \mathrm{~cm}^{-2}$ and $\mu=1.1 \times 10^{7} \mathrm{~cm}^{2} / \mathrm{V} \mathrm{s}$ that shows IQHE up to $i \approx 40$, Fig. 3(a) illustrates $R_{x x}, R_{x y}$, and $\Delta R_{x y}$ for both $B$ directions, using the convention $R_{x y}>0$ for $B>0$. Figures 3(b) and 3(c) confirm similar behavior for both $B$ directions once the antisymmetry in $\Delta R_{x y}$ under $B$ reversal is taken into account. Figure 3(c) indicates that from $20 \leq \nu<46, \Delta R_{x y}$ oscillations are approximately orthogonal to the $\Delta R_{x x}$ oscillations, as in Fig. 1(c). This feature, plus the manifestation of Hall plateaus in Fig. 3(a), and the consistency with the resistivity/resistance rule indicated in Fig. 3(d) over this $\nu$ range, confirms that the IQHE observed here is the canonical effect. A remarkable and interesting feature in Fig. 3(c) is that, following a
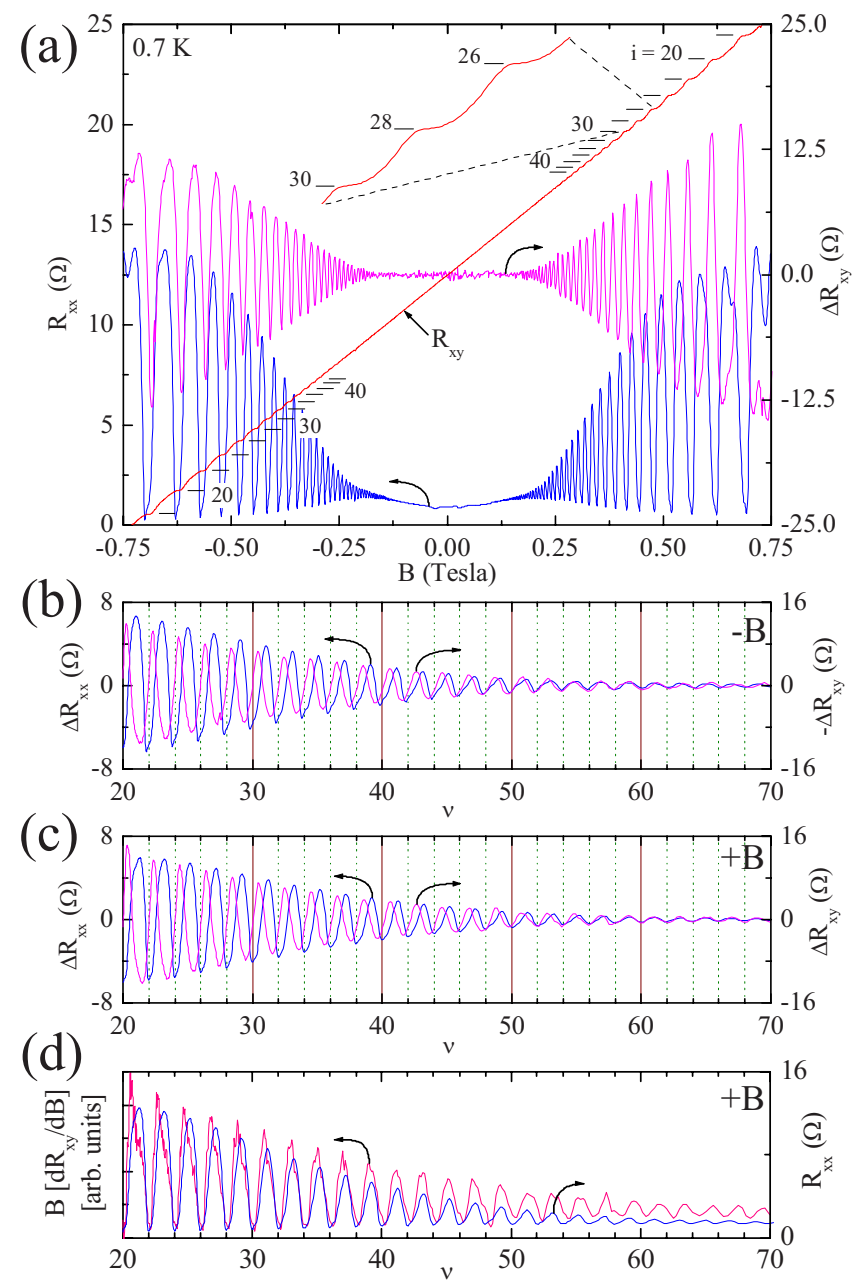

FIG. 3. (Color online) Type 1 to type 2 crossover at large $\nu$ : (a) $R_{x x}, R_{x y}$, and the oscillatory Hall resistance, $\Delta R_{x y}$, are shown over low magnetic fields, $B$, for a high-mobility GaAs/AlGaAs specimen. Here, the oscillatory Hall resistance is antisymmetric under field reversal, i.e., $\Delta R_{x y}(-B)=-\Delta R_{x y}(+B)$, as expected. (b) The oscillatory diagonal resistance $\left(\Delta R_{x x}\right)$ and $\Delta R_{x y}$ have been plotted vs $\nu$ to compare their relative phases for $-B$. (c) As above for $+B$. For $20 \leq \nu<46, \Delta R_{x x}$ and $\Delta R_{x y}$ are approximately orthogonal as in Fig. 1 (c). For $56 \leq \nu \leq 70, \Delta R_{x x}$ and $\Delta R_{x y}$ are approximately in-phase, unlike at $\nu<46$. Note that the right ordinates in Fig. 3(c) and Fig. 3(b) show $+\Delta R_{x y}$ and $-\Delta R_{x y}$, respectively, in order to account for the antisymmetry in $\Delta R_{x y}$ under $B$ reversal. (d) $B\left(d R_{x y} / d B\right)$ and $R_{x x}$ are plotted vs $\nu$. For $\nu<46$, the two quantities are similar and in-phase, while a phase difference develops at higher $\nu$.

smooth crossover, $\Delta R_{x x}$ and $\Delta R_{x y}$ become in-phase, i.e., type 2 , for $\nu \geq 56$, as a variance with the resistivity/resistance rule, in the form of a phase shift, develops in Fig. 3(d).

Figures 4(a) and 4(b) provide further evidence for inphase type 2 oscillations in a Hall bar. Here, the Hall oscillations tend to enhance the magnitude of $R_{x y}$ at the $R_{x x} \mathrm{SdH}$ maxima (type 2), even as Hall plateaus are imperceptible in the $R_{x y}$ curve. Yet, from Fig. 4(a), it is clear that $\Delta R_{x y}$ is a Hall effect component, and not a misalignment offset admixture of $R_{x x}$ into $R_{x y}$, since $\Delta R_{x y}$ is antisymmetric under $B$ reversal. We have presented these data exhibiting these type 2 oscillations and type 1 to type 2 crossover mainly for the 
(a)
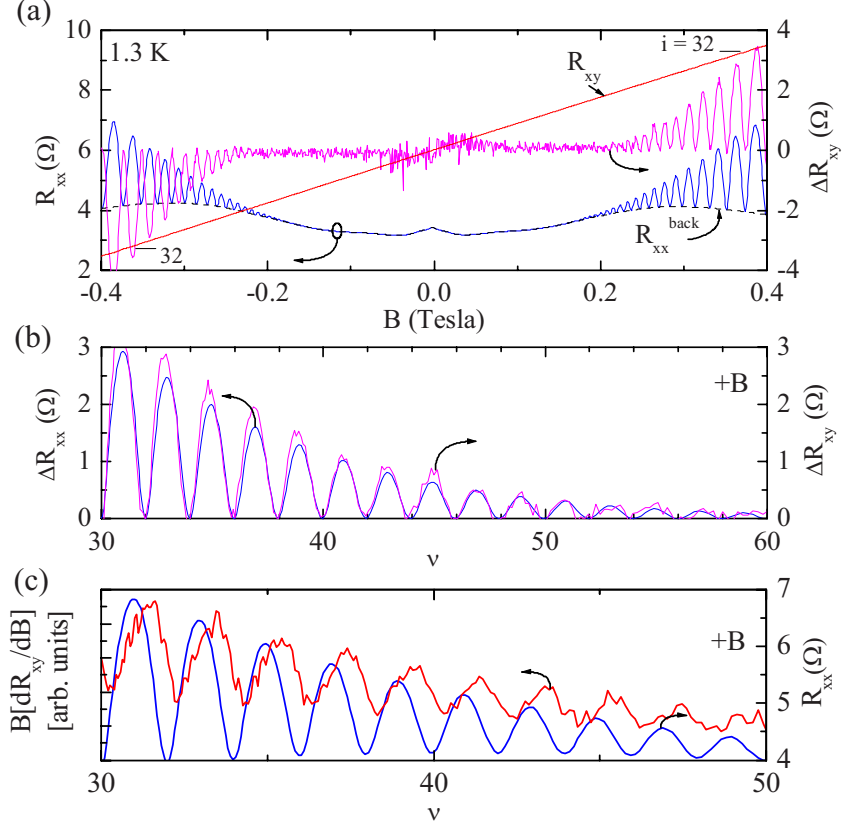

FIG. 4. (Color online) Type 2 oscillations at large $\nu$ : (a) $R_{x x}, R_{x y}$, and $\Delta R_{x y}$ have been shown for a Hall bar specimen with $n=2.9$ $\times 10^{11} \mathrm{~cm}^{-2}$ and $\mu=6 \times 10^{6} \mathrm{~cm}^{2} / \mathrm{V}$ s, which exhibits in-phase $R_{x x}$ and $\Delta R_{x y}$ oscillations. Note the absence of discernible Hall plateaus in $R_{x y}$, when the oscillatory resistances are in-phase. (b) Here, the amplitudes of $\Delta R_{x x}$ and $\Delta R_{x y}$ show similar $\nu$ variation. (c) $B\left(d R_{x y} / d B\right)$ and $R_{x x}$ are plotted vs $\nu$. A comparison of the two traces suggests a $90^{\circ}$ phase shift between the two quantities.

sake of completeness; the main focus of this paper are the results that appear in the following.

\section{Type 3 phase relation at large $\nu$ and an unfamiliar IQHE}

Figure 5 illustrates the third (type 3) phase relation in a high-mobility square shape 2DES with $n=2.9 \times 10^{11} \mathrm{~cm}^{2}$ and $\mu=1 \times 10^{7} \mathrm{~cm}^{2} / \mathrm{V} \mathrm{s}$. Although $\mu$ for this specimen is similar to the one examined in Figs. 3(a)-3(d), the experimental results do look different. Figure 5(a) exhibits data taken at $T=0.85 \mathrm{~K}$ and $T=0.5 \mathrm{~K}$. Figure 5(a) shows that the main effect of changing $T$ is to modify the amplitude of the $\Delta R_{x x}$ and $\Delta R_{x y}$ oscillations, so that oscillatory effects persist to a lower $B$ at the lower $T$. The data of Fig. 5(a) also show that $\Delta R_{x y}$ tends to reduce the magnitude of $R_{x y}$ over the $B$ intervals corresponding to the $R_{x x}$ peaks, as in Fig. 2(c), the type 3 case. Meanwhile, quantum Hall plateaus are easily perceptible in $R_{x y}$; see Fig. 5(a). Figures 5(b) and 5(c) demonstrate that for $+B$, for example, $\Delta R_{x x}$ and $-\Delta R_{x y}$ show nearly the same line shape for $30 \leq \nu \leq 60$, and the phase relation does not change with $T$. Meanwhile, a comparison of $B\left[d R_{x y} / d B\right]$ and $R_{x x}$, see Fig. 5(d), suggests a variance with the resistivity/resistance rule. Indeed, the correlation between the oscillatory diagonal and off-diagonal resistances held true down to nearly $\nu=5$, see left inset of Fig. 5(e), as narrow IQHE plateaus were manifested in $R_{x y}$, see Fig. 5(e). For $\nu$ $\leq 4$, however, $R_{x x}$ correlated better with $B\left[d R_{x y} / d B\right]$, see right inset of Fig. 5(e), than with $-\Delta R_{x y}$, which suggested that the resistivity/resistance rule ${ }^{50}$ might come into play at
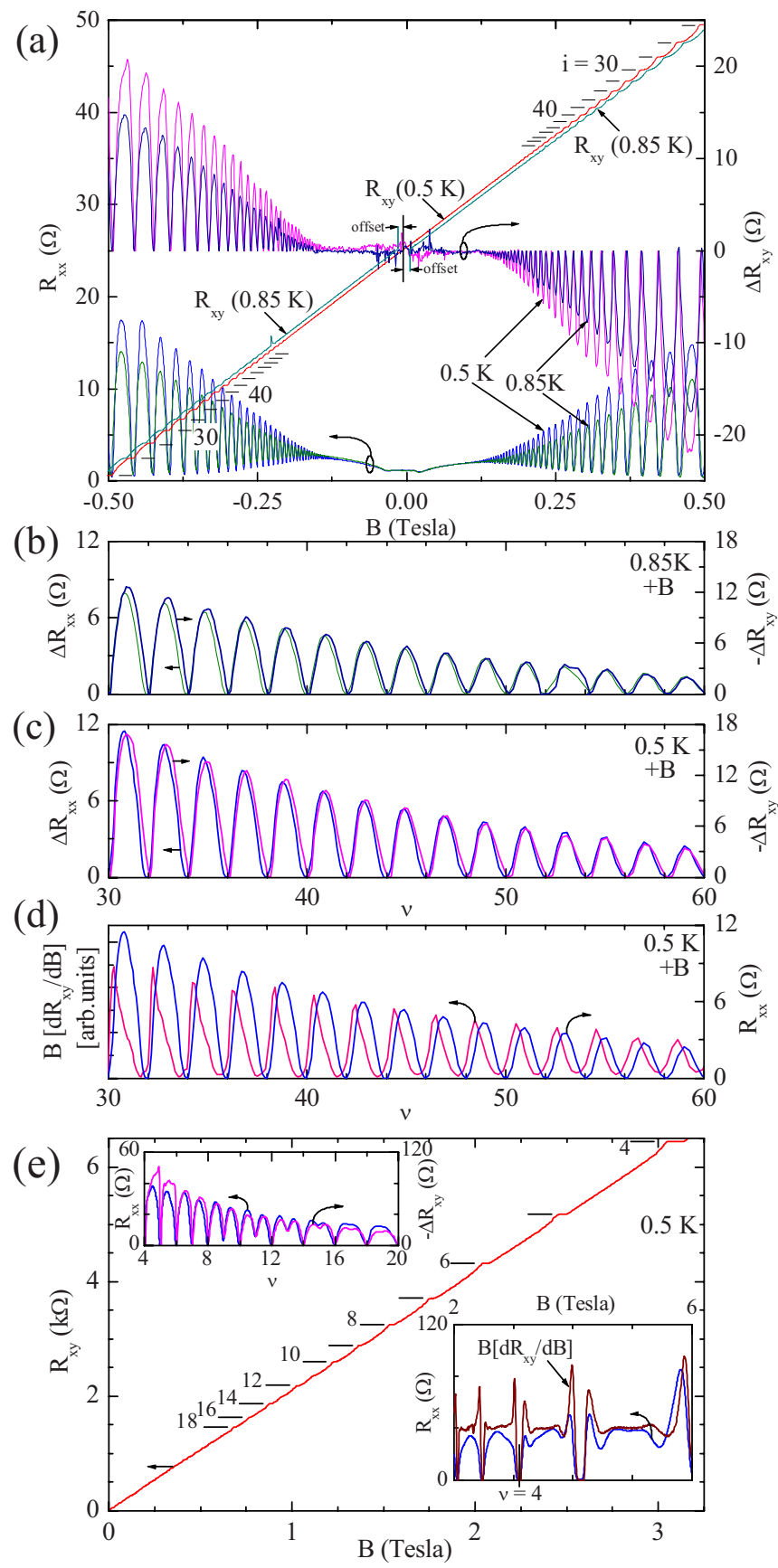

FIG. 5. (Color online) Type 3 oscillations at large $\nu$ and type 3 to type 1 crossover at large $B$ : (a) data for a square shape GaAs/ AlGaAs specimen, where the magnitude of $R_{x y}$ is reduced at the $R_{x x}$ oscillation maxima. Here, the $+B$ and the $-B$ portions of the $R_{x y}(0.85 \mathrm{~K})$ curve have been offset in opposite directions along the abscissa with respect to the $R_{x y}(0.5 \mathrm{~K})$ curve, for the sake of presentation. Note the well-developed plateaus in $R_{x y}$. (b) At $T$ $=0.85 \mathrm{~K}$, a plot of $\Delta R_{x x}$ and $-\Delta R_{x y}$ confirms similarity and a type 3 phase relation. (c) Same as (b) but at $T=0.5 \mathrm{~K}$. (d) $B\left(d R_{x y} / d B\right)$ and $R_{x x}$ are plotted vs $\nu$. The plot shows incommensurability between the two quantities. (e) The main panel shows the Hall resistance vs $B$, with relatively narrow Hall plateaus, down to around filling factor $\nu=4$. Left inset: as in Figs. 5(b) and 5(c) above, $-\Delta R_{x y}$ follows $R_{x x}$, down to nearly $\nu=5$. Right inset: for $\nu \leq 4$, a better correspondence develops between $R_{x x}$ and $B\left[d R_{x y} / d B\right]$. 

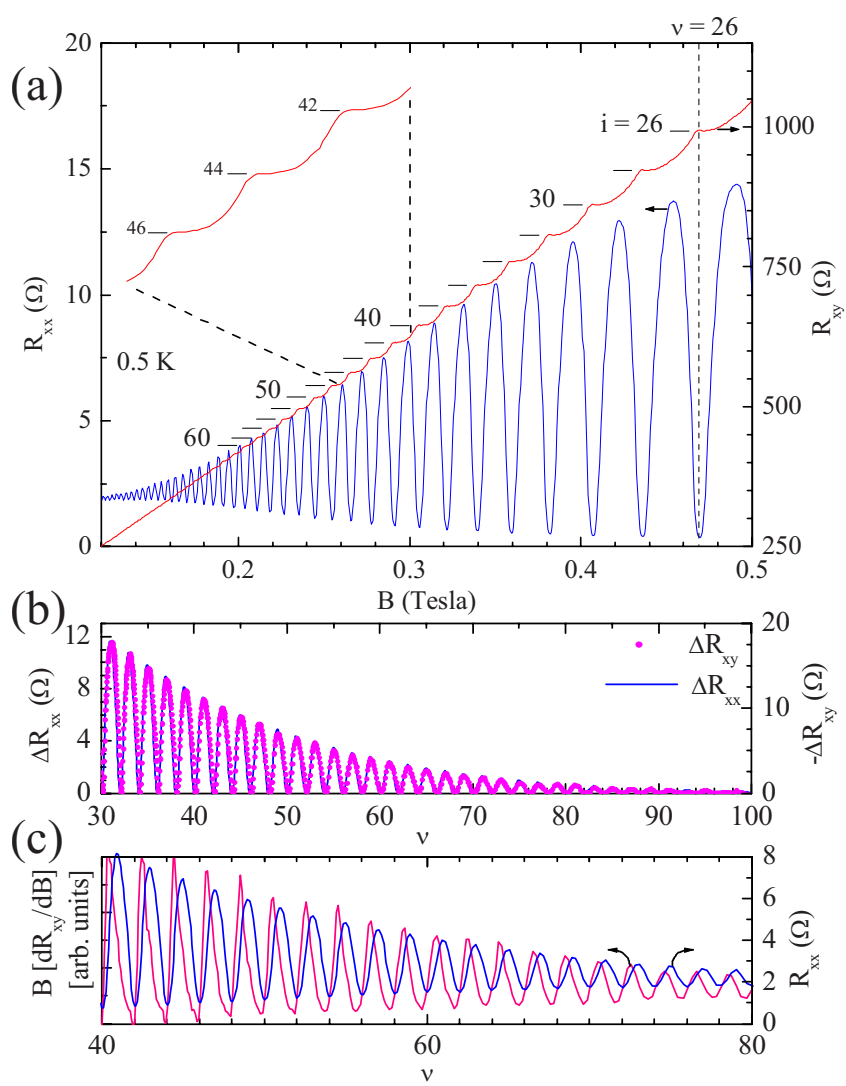

FIG. 6. (Color online) IQHE with type 3 oscillations: (a) $R_{x x}$ and $R_{x y}$ are exhibited for a high-mobility GaAs/AlGaAs specimen that shows deep $R_{x x}$ minima and even-integral Hall plateaus at $R_{x y}$ $=h / i e^{2}$. (b) Here, $-\Delta R_{x y}$ follows $\Delta R_{x x}$, indicative of a $\pi$ phase shift and a type 3 relationship, unlike the canonical quantum Hall situation; see Fig. 1(c). (c) $B\left(d R_{x y} / d B\right)$ and $R_{x x}$ are plotted vs $\nu$ to compare with the resistivity/resistance rule. Here, the line shapes look dissimilar, and there is a phase shift between $B\left(d R_{x y} / d B\right)$ and $R_{x x}$.

especially low $\nu$ here, as the system undergoes a type 3 $\rightarrow$ type 1 transformation, with decreasing $\nu$.

An expanded data plot of type 3 transport is provided in Fig. 6(a). This plot shows plateaus in $R_{x y}$ and deep minima in $R_{x x}$ to very low magnetic fields, as quantum Hall plateaus in $R_{x y}$ follow $R_{x y}=h / i e^{2}$, to an experimental uncertainty of $\approx 1$ percent. Although the IQHE data of Fig. 6(a) again appear normal at first sight, the remarkable difference becomes apparent when $\Delta R_{x x}$ and $-\Delta R_{x y}$ are plotted vs $\nu$, as in Fig. 6(b). Here, we find once again an approximate phase shift of $\pi$ (type 3) between $\Delta R_{x x}$ and $\Delta R_{x y}$ [Fig. 6(b)], as in Figs. 5(b) and $5(\mathrm{c})$, which is distinct from the canonical (type 1) phase relationship exhibited in Fig. 1(c). The resistivity/resistance rule $R_{x x} \propto B\left[d R_{x y} / d B\right]$ seems not to be followed in this case at such large $\nu$, as a "phase shift" and a line shape difference between $R_{x x}$ and $B\left[d R_{x y} / d B\right]$ becomes perceptible in Fig. 6(c). Notably, in Fig. 6(a), the reported type 3 phase relation can even be discerned by a trained eye.

\section{Discussion: Numerical simulations indicating the possibility of type 3 and type 2 oscillations}

It is possible to extract some understanding from the phase relationships observed here between the oscillatory $R_{x x}$

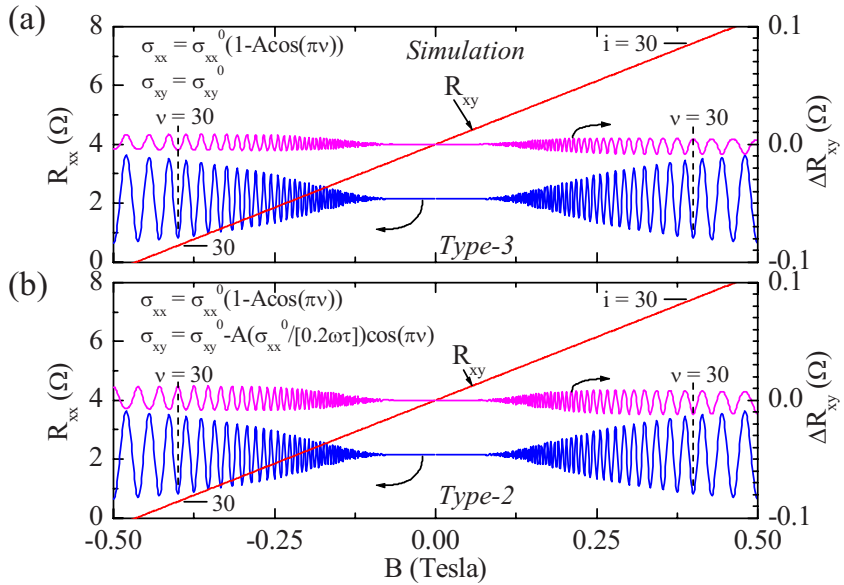

FIG. 7. (Color online) Modeling shows the possibility of type 3 and type 2 oscillations: (a) simulations suggest that oscillatory scattering contributions to the diagonal conductivity alone can produce type 3 oscillations via the tensor relation for the resistivities, where the magnitude of $R_{x y}$ is reduced at the $\mathrm{SdH} R_{x x}$ maxima. (b) Simulations of a semiempirical model that includes both a scattering contribution in $\sigma_{x y}$, and a reduction in the relaxation time with respect to the transport lifetime, indicate the possibility also of type 2 oscillations, with $\left|\Delta R_{x y}\right| \ll\left|\Delta R_{x x}\right|$. A comparison of Figs. 7(a) and 7(b) helps to convey the $\tau$-induced type 3 to type 2 transformation.

(or $\Delta R_{x x}$ ) and $\Delta R_{x y}$. The type 1 orthogonal phase relation of Fig. 1(c) can be viewed as a restatement of the empirical resistivity/resistance rule, since the data of Fig. 1(a) yield both Fig. 1(b) and Fig. 1(c). Theory suggests that this rule might follow when $R_{x x}$ is only weakly dependent on the local diagonal resistivity $\rho_{x x}$ and approximately proportional to the magnitude of fluctuations in the off-diagonal resistivity $\rho_{x y}$, when $\rho_{x x}$ and $\rho_{x y}$ are functions of the position. ${ }^{53}$ Thus, according to theory, specimens following the resistivity rule (and exhibiting type 1 oscillations) seem likely to include density fluctuations. ${ }^{53}$

For type 2 and type 3 oscillations, note that the specimens of Figs. 2-6 satisfy $\omega \tau_{T}>1$, with $\omega$ the cyclotron frequency, and $\tau_{T}$ the transport lifetime, at $B>0.001$ (or 0.002) $T$. One might semiempirically introduce oscillations into the diagonal conductivity, $\sigma_{x x}$, as $\sigma_{x x}=\sigma_{x x}^{0}\left[1-A \cos \left(2 \pi E_{F} / \hbar \omega\right)\right] .{ }^{54-56}$ Here, the minus sign ensures the proper phase, while $\sigma_{x x}^{0}=\sigma_{0} /\left[1+\left(\omega \tau_{T}\right)^{2}\right], \quad \sigma_{0}$ is the dc conductivity, $E_{F}$ is the Fermi energy, and $A=4 c\left\{\left(\omega \tau_{T}\right)^{2} /\left[1+\left(\omega \tau_{T}\right)^{2}\right]\right\}[X / \sinh (X)]$ $\times \exp \left(-\pi / \omega \tau_{S}\right)$, where $X=\left[2 \pi^{2} k_{B} T / \hbar \omega\right], \tau_{S}$ is single particle lifetime, and $c$ is of order unity. ${ }^{54,56,57}$ Simulations with $\sigma_{x x}$ as given above, and $\sigma_{x y}=\sigma_{x y}^{0}=\left(\omega \tau_{T}\right) \sigma_{x x}^{0}$, indicate oscillations in both $R_{x x}$ and $R_{x y}$ via $\rho_{x x}=\sigma_{x x} /\left(\sigma_{x x}^{2}+\sigma_{x y}^{2}\right)$ and $\rho_{x y}$ $=\sigma_{x y} /\left(\sigma_{x x}^{2}+\sigma_{x y}^{2}\right)$, and a type 3 phase relationship, see Fig. 7 (a), with $\left|\Delta R_{x y}\right| \ll\left|\Delta R_{x x}\right|$. That is, an oscillatory $\sigma_{x x}$ can also lead to small $R_{x y}$ oscillations, with type 3 phase characteristics; see Fig. 7(a).

As a next step, one might introduce an oscillatory $\sigma_{x y}=\sigma_{x y}^{0}\left[1+G \cos \left(2 \pi E_{F} / \hbar \omega\right)\right]$, where $G=2 c\left\{\left[1+3\left(\omega \tau_{T}\right)^{2}\right] /\right.$ $\left.\left[\left(\omega \tau_{T}\right)^{2}\left(1+\left(\omega \tau_{T}\right)^{2}\right)\right]\right\}[X / \sinh (X)] \exp \left(-\pi / \omega \tau_{S}\right){ }^{55,56}$ Upon inverting the tensor including oscillatory $\sigma_{x y}$ and $\sigma_{x x}$, type 3 oscillations were still obtained, as in Fig. 7(a).

Finally, the strong $B$ field $\sigma_{x y}$ follows $\sigma_{x y}=\sigma_{x x} / \omega \tau$ $-n e / B$ in the self-consistent Born approximation for short 
range scattering potentials, when $\tau$ is the relaxation time in the $B$ field. ${ }^{55}$ Although, the dominant scattering mechanism is long-ranged in $\mathrm{GaAs} / \mathrm{AlGaAs}$ devices, we set $\sigma_{x y}$ $=\left(\sigma_{x x}^{0} / \omega \tau-n e / B\right)-A\left(\sigma_{x x}^{0} / \omega \tau\right) \cos \left(2 \pi E_{F} / \hbar \omega\right)$. When $\tau=\tau_{T}$, this approach again yielded type 3 phase relations, as in the discussion above. At this point, we were surprised to see that the type 3 oscillations reported here could be so readily generated from the simulations. Next, we examined the case $\tau$ $<\tau_{T}$, in order to account for the possibility that $\tau$ in a $B$ field may possibly come to reflect $\tau_{S}$, which typically satisfies $\tau_{S}$ $<\tau_{T}$ for small angle scattering by long-range scattering potentials. ${ }^{57}$ Remarkably, a reduction in $\tau$, which corresponds to changing the nature of the potential landscape, converted type 3 (phase shift by $\pi$ ) to type 2 (in-phase) oscillations; see Figs. 7(a) and 7(b).

If density fluctuations at large length scales produce type 1 characteristics, ${ }^{53}$ and type 2 oscillations require a difference between $\tau_{T}$ and $\tau$ as suggested above, then the observation of type 1 and type 2 oscillations in the same measurement [Figs. 3(b) and 3(c)] seems consistent because long length-scale potential fluctuations can produce both modest density variations and a difference between $\tau_{T}$ and $\tau_{S}$ (or $\tau){ }^{57}$ Perhaps, with increasing $B$, there is a crossover from type 2 to type 1 before $R_{x y}$ plateaus become manifested, and thus, IQHE is not indicated in the type 2 regime; see Figs. 3(a) and 4(a).

Specimens exhibiting type 3 oscillations and associated IQHE suggest better homogeneity in $n$, which is confirmed by oscillations to extremely low $B$ [see Figs. 6(a) and 6(b)]. The relatively narrow plateaus at high $B$ [see Fig. 5(e)] hint at a reduced role for disorder-induced localization. ${ }^{2}$ In this case, perhaps there are other mechanisms contributing to the observed large $\left|\Delta R_{x y}\right|$, and plateau formation, in the type 3 case in the high- $\mu$ system. It could be that such additional mechanisms serve to create, maintain, or enhance the mobility gap or suppress backscattering in the higher Landau level here, which assists in the realization of the type 3 characteristics and associated unfamiliar IQHE to low $B$.

\section{PART 2: TRANSPORT STUDY UNDER MICROWAVE PHOTOEXCITATION}

Previous sections indicated the observability of several possible phase relations, denoted as type 1 , type 2 , and type 3 , between the oscillatory Hall and diagonal resistances in the dark-GaAs/AlGaAs system. Of particular interest were the approximately $\pi$-shifted type 3 oscillations (Figs. 5 and 6 ), which exhibited quantum Hall effects that were similar to-and yet different from-the canonical quantum Hall effect that goes together with the resistivity/resistance rule and type 1 oscillations (Fig. 1). Numerical simulations exhibited in the last section confirmed the possibility of these type 3 and type 2 phase relations, although the magnitude of the Hall oscillations obtained in the simulations always remained small compared to $R_{x x}$ oscillations, unlike experiment. In this section, we examine the influence of microwave photoexcitation over the same magnetic field, filling factor, and temperature intervals, to determine - and convey - the radiationinduced change in Shubnikov-de Haas oscillations and

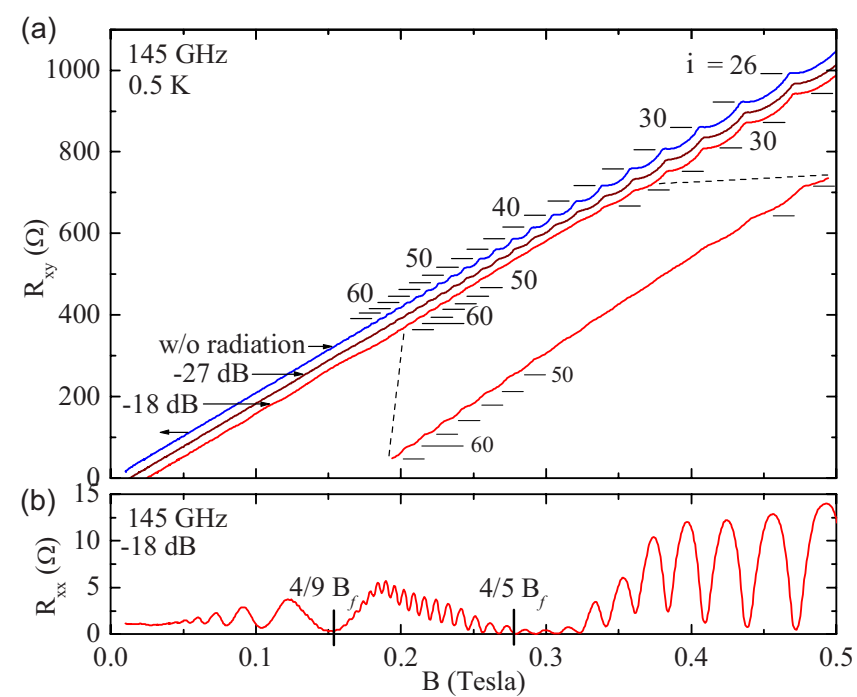

FIG. 8. (Color online) Photoexcitation eliminates IQHE: (a) the dark- and irradiated-at-145 GHz-off-diagonal Hall resistances $R_{x y}$ of a GaAs/AlGaAs device at $T=0.5 \mathrm{~K}$ have been exhibited vs the magnetic field $B$. For the sake of presentation, the $-27 \mathrm{~dB}$ and $-18 \mathrm{~dB}$ curves have been shifted down along the ordinate with respect to the dark "w/o radiation" curve. The index, $i$, labels the Hall plateaus. Also shown in the figure panel is an expanded $R_{x y}$ plot of the $-18 \mathrm{~dB}$ curve for the span $34 \leq i \leq 62$, which shows that Hall plateaus disappear under photoexcitation between roughly 36 $\leq i \leq 48$. Thus, photoexcitation eliminates these quantum Hall effects in favor of an ordinary Hall effect. (b) This panel exhibits the diagonal resistance $R_{x x}$ under photoexcitation at $145 \mathrm{GHz}$, with the photoexcitation attenuated to $-18 \mathrm{~dB}$. Note the reduction in the amplitude of Shubnikov-de Haas oscillations, most noticeably in the vicinity of the $(4 / 5) B_{f}$ minimum of the radiation-induced magnetoresistance oscillations. Thus, this figure shows that a radiationinduced reduction in the amplitude of Shubnikov-de Haas oscillations in $R_{x x}$ correlates with the radiation-induced disappearance of IQHE plateaus in $R_{x y}$.

IQHE in the type 3 high-mobility system. The MBE grown GaAs/AlGaAs single heterostructures for these measurements were prepared by Umansky and co-workers, as per Ref. 58.

Figure 8(a) illustrates the dark and irradiated-at-145 GHz off-diagonal Hall resistances $R_{x y}$ of a GaAs/AlGaAs device at $T=0.5 \mathrm{~K}$. Here, for the sake of presentation, the $-27 \mathrm{~dB}$ and $-18 \mathrm{~dB}$ Hall curves have been downshifted along the ordinate with respect to the dark "w/o radiation" Hall curve, and the Hall plateaus have been labeled with the index, $i$. Figure 8 (a) shows that the $R_{x y}$ curve is influenced by the radiation in two ways. First, there develops the "slow" radiation-induced oscillations in the Hall resistance, which are superimposed on the overall linear increase in $R_{x y}$ with $B$. These radiation-induced oscillations in $R_{x y}$ are especially evident in the $-18 \mathrm{~dB}$ curve, and they are similar to what we have reported earlier. ${ }^{9}$ Second, radiation also appears to narrow the width of the Hall plateaus more readily over some range of filling factors than others. Again, this feature is most evident in the $-18 \mathrm{~dB}$ curve. Indeed, in the expanded $R_{x y}$ plot of the $-18 \mathrm{~dB}$ curve in Fig. 8(a) for the span $34 \leq i$ $\leq 62$, the Hall plateaus tend to vanish under photoexcitation 


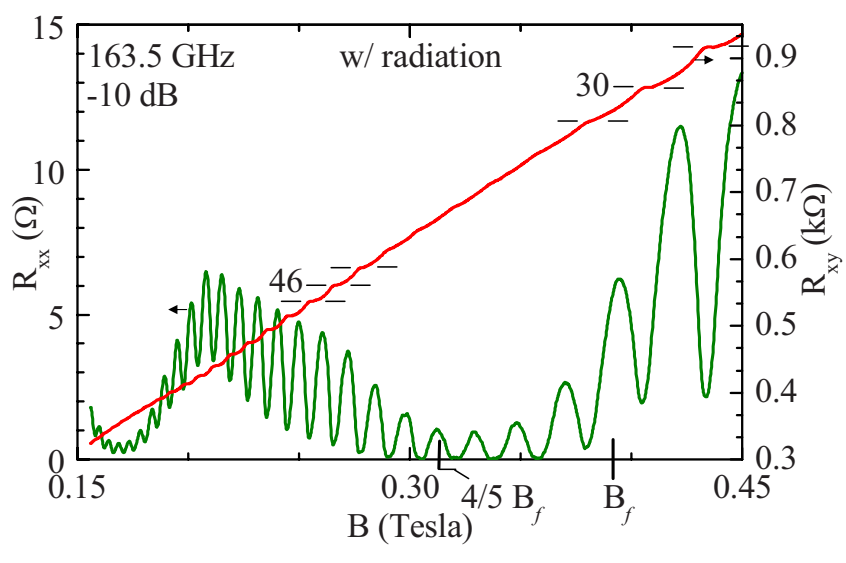

FIG. 9. (Color online) Re-entrant IQHE under photoexcitation at $T=0.5 \mathrm{~K}$ : the diagonal $\left(R_{x x}\right)$ and off-diagonal $\left(R_{x y}\right)$ resistances of a photoexcited GaAs/AlGaAs device have been exhibited vs the magnetic field, $B$, for $0.15 \leq B \leq 0.45 \mathrm{~T}$. Here, the specimen has been photoexcited at $163.5 \mathrm{GHz}$, with the intensity attenuated to $-10 \mathrm{~dB}$. The "slow" oscillatory structure in $R_{x x}$ corresponds to the radiation-induced magnetoresistance oscillations, while the "fast" structure corresponds to the Shubnikov-de Haas oscillations. The $(4 / 5) B_{f}$ minimum for the radiation-induced magnetoresistance oscillations has been marked in the figure. Noticeably, the amplitude of the Shubnikov-de Haas oscillations in $R_{x x}$ is reduced in the vicinity of the minima of the radiation-induced magnetoresistance oscillations, and this feature correlates with a vanishing of IQHE in $R_{x y}$ over the same interval. Specifically, in this instance, Hall plateaus vanish between $34 \leq i \leq 42$. They reappear at higher $i$, only to disappear once again at even higher $i$. That is, there appears to be a radiation-induced re-entrance into IQHE. Here, the plateau index $i$ has been marked next to the Hall plateaus.

between roughly $36 \leq i \leq 48$, only to reappear at even higher $i$. Thus, this figure suggests that photoexcitation exchanges the integral quantum Hall effects with an ordinary Hall effect over some range of filling factors. The bottom panel of Fig. 8 exhibits the diagonal resistance $R_{x x}$ under photoexcitation at $f=145 \mathrm{GHz}$, with the photoexcitation attenuated to $-18 \mathrm{~dB}$. This panel exhibits strong radiation-induced magnetoresistance oscillations in $R_{x x}$, and a reduction in the amplitude of Shubnikov-de Haas oscillations, most noticeably in the vicinity of the $(4 / 5) B_{f}$ minimum, where $B_{f}=2 \pi m^{*} f / e$. A comparison of the top and bottom panels of Fig. 8 indicates that the radiation-induced vanishing of Hall plateaus in $R_{x y}$ correlates with this radiation-induced nonmonotonicity in the amplitude of Shubnikov-de Haas oscillations in $R_{x x}$.

Figure 9 exhibits similar characteristics at a radiation frequency $f=163.5 \mathrm{GHz}$. Here, a simultaneous measurement of $R_{x x}$ and $R_{x y}$, followed by a plot of the two curves on the same graph, helps to convey the intimate relation between the radiation-induced nonmonotonic variation in the amplitude of the Shubnikov-de Haas $R_{x x}$ oscillations and the nonmonotonic variation in the width of the Hall plateaus in $R_{x y}$. This plot suggests that as the amplitude of Shubnikov-de Haas oscillations in $R_{x x}$ is reduced by photoexcitation, for example, in the vicinity of the $(4 / 5) B_{f}$ radiation-induced resistance minimum, the Hall plateaus tend to narrow and vanish, although the plateaus reappear once again at either end of the $(4 / 5) B_{f}$ minimum, when the Shubnikov-de Haas oscillations in $R_{x x}$ grow stronger.
Figure 10 helps to convey the evolution of these transport characteristics with the photoexcitation intensity at $f$ $=130 \mathrm{GHz}$. Hence, Fig. 10 (a) shows $R_{x x}$ and $R_{x y}$ vs $B$ with the radiation attenuated to $-13 \mathrm{~dB}$, while Fig. 10(b) shows the same with the radiation attenuated to $-8 \mathrm{~dB}$. Here, Fig. 10(b) corresponds to the more intense photoexcitation. Although the two panels look similar, it is apparent that increasing the intensity from $-13 \mathrm{db}$ to $-8 \mathrm{~dB}$ increases the magnitude of the "slow" radiation-induced magnetoresistance oscillations characterized by relatively broad minima in the vicinity of $(4 / 5) B_{f}$ and $(4 / 9) B_{f}$. Concurrently, the amplitude of Shubnikov-de Haas oscillations in $R_{x x}$ becomes smaller in the vicinity of, for example, the $(4 / 5) B_{f}$ minimum. This weakening of $\mathrm{SdH}$ oscillations goes together again with the progressive disappearance of IQHE. It is worth pointing out that IQHE were easily observable down to approximately $0.2 \mathrm{~T}$ at this temperature, $T=0.5 \mathrm{~K}$, in the absence of photoexcitation, as in Fig. 6. Figure 10 shows, however, that under photoexcitation, although some IQHE are still observable in the vicinity of $0.2 \mathrm{~T}$, they disappear in the vicinity of $0.25 \mathrm{~T}$ as a consequence of the photoexcitation, only to reappear at an even higher $B$.

The data of Figs. 8-10 help to illustrate that microwave photoexcitation not only produces radiation-induced magnetoresistance oscillations, but it also influences the amplitude of Shubnikov-de Haas oscillations, producing a nonmonotonic variation in the amplitude of these oscillations vs $B$ or $\nu$. Further, the data show that this nonmonotonic variation in the amplitude of the Shubnikov-de Haas oscillations correlates with a nonmonotonic variation in the width of the IQHE plateaus vs $B$ or $\nu$. As a vanishing Hall plateau width signals the disappearance of IQHE, it appears that photoexcitation helps to replace the IQHE with an ordinary Hall effect over the minima of the radiation-induced magnetoresistance oscillations.

In order to identify the mechanism responsible for the disappearance of the Hall plateaus under photoexcitation, it seems necessary to establish first the nature of IQHE at these high filling factors per the analysis used earlier for examining transport in the dark specimens. Hence, in Fig. 11, we replot the data of Fig. 10 as $R_{x x}$ vs $\nu$ and $\Delta R_{x y}$ vs $\nu$. The plots of Fig. 11 are generally similar to the plots of Fig. 1(c), Figs. 3(b) and 3(c), Fig. 4(b), Figs. 5(b) and 5(c), and Fig. 6(b), with the difference that, instead of $\Delta R_{x x}$, it is $R_{x x}$ that is directly compared with $-\Delta R_{x y}$, in the plot overlays. The reason for this difference is that we wish to compare, at the same time, the relative phases of the "fast" "SdH oscillations," as well as the "slow" radiation-induced resistance oscillations, in $R_{x x}$ and $R_{x y}$.

In previous work, ${ }^{9}$ we have shown that so far as the radiation-induced oscillations in $R_{x x}$ and $R_{x y}$ are concerned, $-\Delta R_{x y} \propto R_{x x}$. By setting up this same relation in the plots of Fig. 11, we notice that both the radiation-induced and "SdH" oscillations in the diagonal and off-diagonal resistances match up over nearly the entire range of exhibited filling factors, $12 \leq \nu \leq 50$. Thus, Fig. 11 helps to realize three conclusions: (a) there is an approximately $\pi$-phase shift between the Shubnikov-de Haas oscillations of the diagonal and off-diagonal Hall resistances in these photoexcited data, as for the type 3 resistance oscillations sketched in Fig. 2(c). 

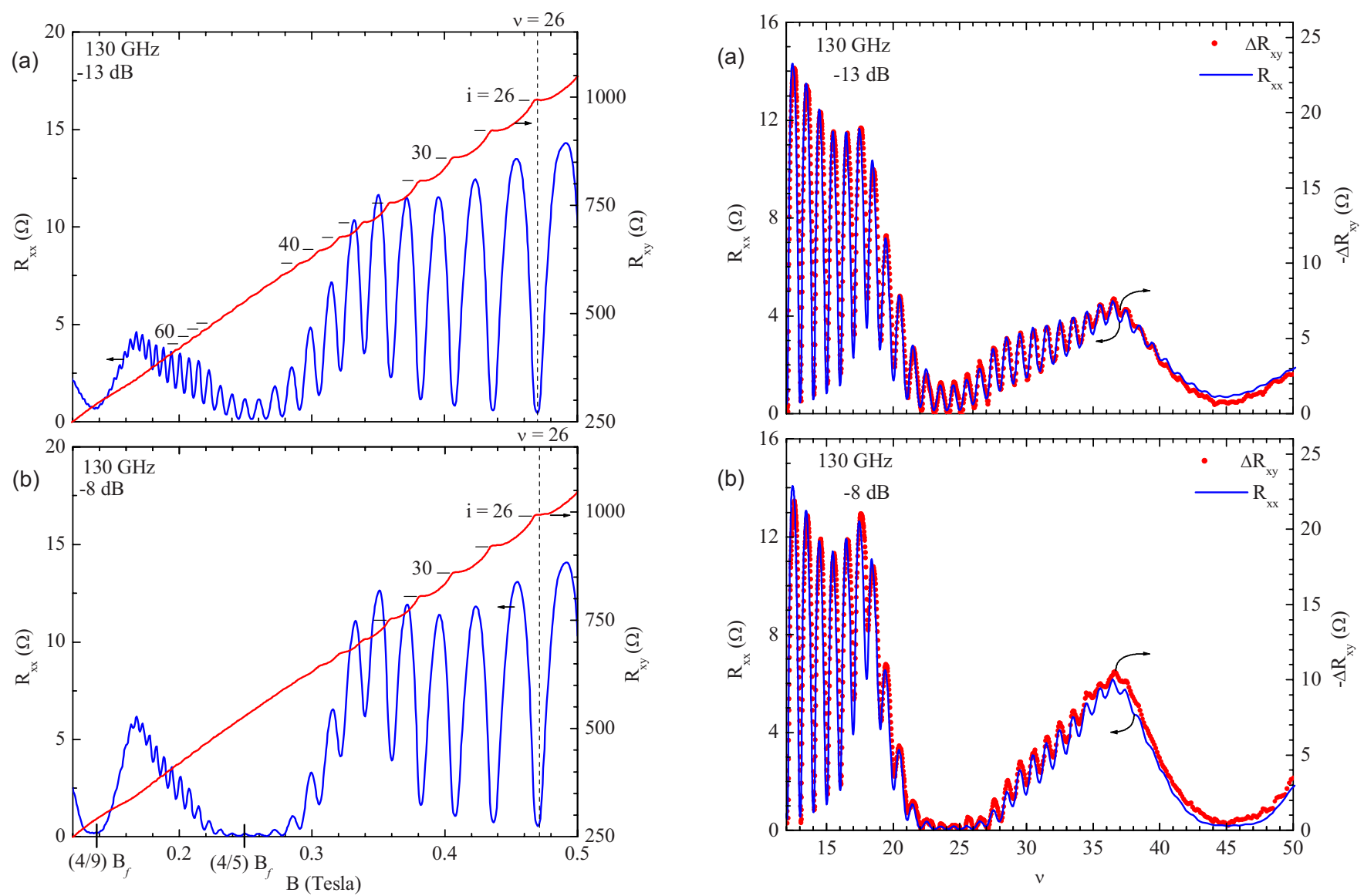

FIG. 10. (Color online) Evolution of transport and re-entrant IQHE under photoexcitation at $T=0.5 \mathrm{~K}$ : the diagonal $\left(R_{x x}\right)$ and off-diagonal $\left(R_{x y}\right)$ resistances of a photoexcited GaAs/AlGaAs device have been exhibited vs the magnetic field, $B$, for $0.12 \leq B$ $\leq 0.5 \mathrm{~T}$. Here, the specimen has been photoexcited at $130 \mathrm{GHz}$, with the intensity attenuated to $-13 \mathrm{~dB}$ in panel (a) and $-8 \mathrm{~dB}$ in panel (b). Thus, a comparison of panels (a) and (b) serves to convey the effect of an incremental change in the radiation intensity. The bottom panel corresponds to the greater intensity. As the radiation intensity increases, the radiation-induced magnetoresistance oscillations in $R_{x x}$, i.e., the "slow" oscillations, become more pronounced and a radiation-induced zero-resistance state becomes perceptible in $R_{x x}$ in the vicinity of $(4 / 5) B_{f}$. Concurrently, the amplitude Shubnikov-de Haas oscillations in $R_{x x}$ show even stronger nonmonotonicity vs $B$. Indeed, the Shubnikov-de Haas oscillations nearly vanish in the vicinity of $(4 / 5) B_{f}$ as a consequence of photoexcitation at $-8 \mathrm{~dB}$. A comparison of the top and bottom panels also shows that Hall plateaus become weaker and tend to vanish with increased photoexcitation near the minima of the radiationinduced magnetoresistance oscillations. The effect tracks the disappearance of Shubnikov-de Haas oscillations in $R_{x x}$ under the influence of photoexcitation. Indeed, some Hall plateaus that are observable under photoexcitation at $-13 \mathrm{~dB}$ seem to have nearly vanished under irradiation at $-8 \mathrm{~dB}$.

(b) Radiation-induced magnetoresistance oscillations of the diagonal and off-diagonal resistances follow the (type 3) relation $-\Delta R_{x y} \propto R_{x x}$, as previously reported. ${ }^{9}$ (c) The IQHE observed in this photoexcited study go together with the type 3 characteristics, cf. Figs. 10 and 11, once again, as in the dark study, cf. Figs. 5 and 6. Thus, the observed IQHE seem

FIG. 11. (Color online) Type 3 transport under photoexcitation: this figure presents a replot of the data of the previous figure, Fig. 10 , as $R_{x x}$ vs $\nu$ and $-\Delta R_{x y}$ vs $\nu$, in order to carry out a phase comparison of the oscillations in the diagonal and off-diagonal resistance. Here, the oscillatory part of the Hall resistance, $\Delta R_{x y}$, matches, within a scale factor, the diagonal resistance so far as both the radiation-induced and Shubnikov-de Haas oscillations are concerned. Indeed, the type 3 behavior observed in this figure confirms that the IQHE exhibited in the previous figure, Fig. 10, corresponds to the new IQHE, discussed in association with Figs. 5 and 6.

to belong once again to a previously unknown class of IQHE.

These strong correlations between the $R_{x x}$ and $-\Delta R_{x y}$ characteristics per Fig. 11 further suggest that the mechanism that is responsible for the radiation-induced modulation in the amplitude of Shubnikov-de Haas oscillations in $R_{x x}$ is also the mechanism that produces the modulation in the width of the IQHE. This topic will be examined in greater detail elsewhere. ${ }^{59}$

Finally, the observation of vanishing diagonal resistance and an ordinary Hall effect under photoexcitation, over a range of filling factors where vanishing resistance is associated with quantum Hall effect in the dark specimen, suggests that the disordered 2D system attains the appearance of an ideal, disorder-free, two-dimensional electron system, under photoexcitation. $^{2}$

\section{CONCLUSION}

An experimental study of the high-mobility GaAs/ AlGaAs system in the dark at large $\nu$ indicates three distinct 
phase relations between the oscillatory Hall and diagonal resistances, which have been labeled as type 1 , type 2 , and type 3 oscillations. Type 1 corresponds to the canonical quantum Hall situation at large filling factors. Type 2 and type 3 oscillations can be reproduced in simple transport simulations and such oscillations exhibit systematic deviations from the resistivity/resistance rule. Surprisingly, IQHE appears manifested in high-mobility specimens in the case of type 3 oscillations, which are characterized by approximately "antiphase" Hall- and diagonal-resistance oscillations. Based on the differences at large $\nu$ between IQHE in the type 3 case and the canonical IQHE, so far as the phase relations and consistency with the resistivity/resistance rule are concerned, we have reasoned the type 3 case corresponds to an unfamiliar and distinct class of IQHE.

We have also examined the influence of photoexcitation at microwave frequencies on the Hall and diagonal resistances in the high-mobility specimen at large filling factors. We have observed that photoexcitation serves to produce a nonmonotonic variation with $B$ or $\nu$ in the amplitude of Shubnikov-de Haas oscillations, and concurrently modulates the width of the IQHE plateaus, leading, remarkably, to a vanishing of IQHE at the minima of the radiation-induced magnetoresistance oscillations. Strikingly, the type 3 phase relation is observed for both the "SdH" oscillations and the radiation-induced magnetoresistance oscillations in $R_{x x}$ and $R_{x y}$; see Fig. 11. The results suggest that the mechanism that produces the modulation in the amplitude of $\mathrm{SdH}$ oscillations in $R_{x x}$ is also responsible for plateau narrowing and IQHE quenching at the minima of the radiation-induced magnetoresistance oscillations.

\section{ACKNOWLEDGMENT}

R.G.M. was supported by D. Woolard and the Army Research Office under Grant No. W911NF-07-01-0158.

\section{APPENDIX: BACKGROUND SUBTRACTION AND THE EXTRACTION OF THE OSCILLATORY RESISTANCES}

The purpose of this appendix is to exhibit the background subtraction procedure that has been used to extract the oscillatory resistances and generate the resistance oscillations overlays in the figures, for phase comparison. For the diagonal resistance, $R_{x x}^{\text {back}}$, when utilized, typically followed either the midpoints [e.g., Fig. 1(a)] or the minima [e.g., Fig.

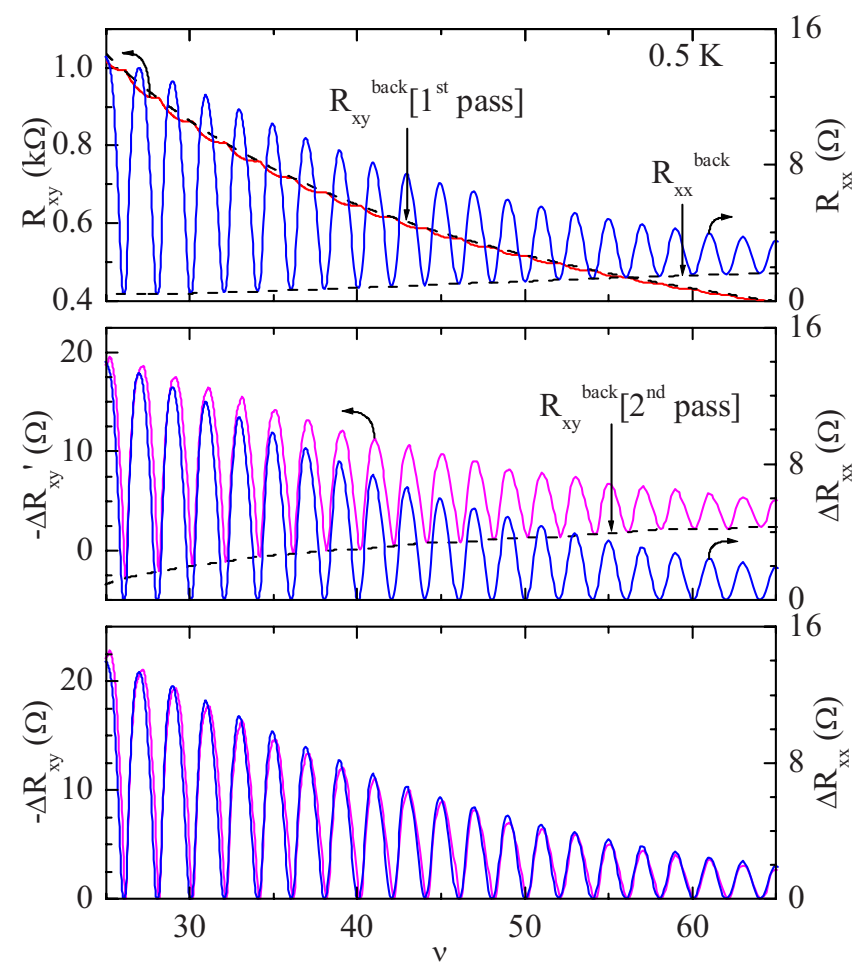

FIG. 12. (Color online) Background subtraction procedure: (top) the diagonal and off-diagonal resistances $R_{x x}$ and $R_{x y}$ of a GaAs/ AlGaAs device have been exhibited vs the filling factor $\nu$ along with $R_{x x}^{\text {back }}$ and $R_{x y}^{\text {back }}$ (first pass), the background Hall resistance at the first pass. $R_{x y}^{\text {back }}$ (first pass) is obtained through a linear fit of $R_{x y}$ vs $B$. (Center) This panel exhibits $\Delta R_{x x}=R_{x x}-R_{x x}^{\text {back }}$ and $-\Delta R_{x y}^{\prime}=-\left(R_{x y}-R_{x y}^{\mathrm{back}}\right)$ (first pass). (Bottom) This panel exhibits $\Delta R_{x x}$ and $-\Delta R_{x y}=-\left(\Delta R_{x y}^{\prime}-R_{x y}^{\text {back }}\right)$ (second pass).

4(a) ] of the $R_{x x}$ oscillations; see also Fig. 12 (top). The resulting $R_{x x}^{\text {back }}$ is then removed from $R_{x x}$ to obtain $\Delta R_{x x}$ as shown in Fig. 12 (center). Background subtraction for the off-diagonal Hall resistance involved a two pass process, since $\left|R_{x y}^{\text {back }}\right| \gg\left|\Delta R_{x y}\right|$. Here, the first pass identified $\approx 99 \%$ of $R_{x y}^{\text {back }}$ through a linear fit of $R_{x y}$ vs $B$ which is shown in Fig. 12 (top), while a spline fit in the second pass, see Fig. 12 (center), then accounted for the $\approx 1 \%$ residual term. At the second pass, $R_{x y}^{\text {back }}$ was chosen as for $R_{x x}$, see above, to make possible resistance oscillations overlays of the type shown in Fig. 12 (bottom). In all cases, $R_{x x}^{\text {back }}$ and $R_{x y}^{\text {back }}$ varied "slowly" in comparison to the oscillatory part of the resistances.
${ }^{1}$ K. v. Klitzing, G. Dorda, and M. Pepper, Phys. Rev. Lett. 45, 494 (1980).

${ }^{2}$ R. E. Prange and S. M. Girvin, The Quantum Hall Effect, 2nd ed. (Springer, New York, 1990).

${ }^{3}$ S. Das Sarma and A. Pinczuk, Perspectives in Quantum Hall Effects (Wiley, New York, 1996).

${ }^{4}$ M. P. Lilly, K. B. Cooper, J. P. Eisenstein, L. N. Pfeiffer, and K. W. West, Phys. Rev. Lett. 82, 394 (1999); J. P. Eisenstein, K. B.
Cooper, L. N. Pfeiffer, and K. W. West, ibid. 88, 076801 (2002).

${ }_{5}^{5}$ J. S. Xia, W. Pan, C. L. Vincente, E. D. Adams, N. S. Sullivan, H. L. Stormer, D. C. Tsui, L. N. Pfeiffer, K. W. Baldwin, and K. W. West, Phys. Rev. Lett. 93, 176809 (2004).

${ }^{6}$ R. G. Mani, J. H. Smet, K. von Klitzing, V. Narayanamurti, W. B. Johnson, and V. Umansky, Nature (London) 420, 646 (2002).

${ }^{7}$ M. A. Zudov, R. R. Du, L. N. Pfeiffer, and K. W. West, Phys. Rev. Lett. 90, 046807 (2003). 
${ }^{8}$ S. I. Dorozhkin, JETP Lett. 77, 577 (2003).

${ }^{9}$ R. G. Mani, V. Narayanamurti, K. von Klitzing, J. H. Smet, W. B. Johnson, and V. Umansky, Phys. Rev. B 69, 161306(R) (2004).

${ }^{10}$ R. G. Mani, J. H. Smet, K. von Klitzing, V. Narayanamurti, W. B. Johnson, and V. Umansky, Phys. Rev. B 69, 193304 (2004).

${ }^{11}$ R. G. Mani, V. Narayanamurti, K. von Klitzing, J. H. Smet, W. B. Johnson, and V. Umansky, Phys. Rev. B 70, 155310 (2004).

${ }^{12}$ R. G. Mani, J. H. Smet, K. von Klitzing, V. Narayanamurti, W. B. Johnson, and V. Umansky, Phys. Rev. Lett. 92, 146801 (2004).

${ }^{13}$ S. A. Studenikin, M. Potemski, P. T. Coleridge, A. S. Sachradja, and Z. P. Wasilewski, Solid State Commun. 129, 341 (2004).

${ }^{14}$ I. V. Kukushkin, M. Y. Akimov, J. H. Smet, S. A. Mikhailov, K. von Klitzing, I. L. Aleiner, and V. I. Falko, Phys. Rev. Lett. 92, 236803 (2004).

${ }^{15}$ R. L. Willett, L. N. Pfeiffer, and K. W. West, Phys. Rev. Lett. 93, 026804 (2004).

${ }^{16}$ R. G. Mani, Physica E (Amsterdam) 22, 1 (2004); 25, 189 (2004).

${ }^{17}$ R. G. Mani, Int. J. Mod. Phys. B 18, 3473 (2004).

${ }^{18}$ R. G. Mani, IEEE Trans. Nanotechnol. 4, 27 (2005).

${ }^{19}$ R. G. Mani, Appl. Phys. Lett. 85, 4962 (2004).

${ }^{20}$ R. G. Mani, Phys. Rev. B 72, 075327 (2005).

${ }^{21}$ J. H. Smet, B. Gorshunov, C. Jiang, L. Pfeiffer, K. West, V. Umanksy, M. Dressel, R. Meisels, F. Kuchar, and K. von Klitzing, Phys. Rev. Lett. 95, 116804 (2005).

${ }^{22}$ Z. Q. Yuan, C. L. Yang, R. R. Du, L. N. Pfeiffer, and K. W. West, Phys. Rev. B 74, 075313 (2006).

${ }^{23}$ R. G. Mani, Solid State Commun. 144, 409 (2007).

${ }^{24}$ R. G. Mani, Appl. Phys. Lett. 91, 132103 (2007).

${ }^{25}$ S. A. Studenikin, A. S. Sachrajda, J. A. Gupta, Z. R. Wasilewski, O. M. Fedorych, M. Byszewski, D. K. Maude, M. Potemski, M. Hilke, K. W. West, and L. N. Pfeiffer, Phys. Rev. B 76, 165321 (2007).

${ }^{26}$ K. Stone, C. L. Yang, Z. Q. Yuan, R. R. Du, L. N. Pfeiffer, and K. W. West, Phys. Rev. B 76, 153306 (2007).

${ }^{27}$ A. Wirthmann, B. D. McCombe, D. Heitmann, S. Holland, K. J. Friedland, and C. M. Hu, Phys. Rev. B 76, 195315 (2007).

${ }^{28}$ R. G. Mani, Physica E 40, 1178 (2008).

${ }^{29}$ R. G. Mani, Appl. Phys. Lett. 92, 102107 (2008).

${ }^{30}$ A. T. Hatke, H. S. Chiang, M. A. Zudov, L. N. Pfeiffer, and K. W. West, Phys. Rev. B 77, 201304(R) (2008).
${ }^{31}$ A. C. Durst, S. Sachdev, N. Read, and S. M. Girvin, Phys. Rev. Lett. 91, 086803 (2003).

${ }^{32}$ A. V. Andreev, I. L. Aleiner, and A. J. Millis, Phys. Rev. Lett. 91, 056803 (2003).

${ }^{33}$ X. L. Lei and S. Y. Liu, Phys. Rev. Lett. 91, 226805 (2003).

${ }^{34}$ V. Ryzhii and A. Satou, J. Phys. Soc. Jpn. 72, 2718 (2003).

${ }^{35}$ P. H. Rivera and P. A. Schulz, Phys. Rev. B 70, 075314 (2004).

${ }^{36}$ S. A. Mikhailov, Phys. Rev. B 70, 165311 (2004).

${ }^{37}$ I. A. Dmitriev, M. G. Vavilov, I. L. Aleiner, A. D. Mirlin, and D. G. Polyakov, Phys. Rev. B 71, 115316 (2005).

${ }^{38}$ J. Inarrea and G. Platero, Phys. Rev. Lett. 94, 016806 (2005).

${ }^{39}$ I. A. Dmitriev, M. G. Vavilov, I. L. Aleiner, A. D. Mirlin, and D. G. Polyakov, Phys. Rev. B 71, 115316 (2005).

${ }^{40}$ J. Inarrea and G. Platero, Phys. Rev. Lett. 94, 016806 (2005).

${ }^{41}$ J. Inarrea and G. Platero, Appl. Phys. Lett. 89, 052109 (2006).

${ }^{42}$ X. L. Lei and S. Y. Liu, Phys. Rev. B 72, 075345 (2005); X. L. Lei, ibid. 73, 235322 (2006).

${ }^{43}$ J. Inarrea, Appl. Phys. Lett. 90, 172118 (2007).

${ }^{44}$ A. D. Chepelianskii, A. S. Pikovsky, and D. L. Shepelyansky, Eur. Phys. J. B 60, 225 (2007).

${ }^{45}$ A. Auerbach and G. V. Pai, Phys. Rev. B 76, 205318 (2007).

${ }^{46}$ I. A. Dmitriev, A. D. Mirlin, and D. G. Polyakov, Phys. Rev. B 75, 245320 (2007).

${ }^{47}$ S. S. Wang and T. K. Ng, Phys. Rev. B 77, 165324 (2008).

${ }^{48}$ J. Inarrea and G. Platero, Phys. Rev. B 78, 193310 (2008).

${ }^{49}$ I. Dmitriev, A. Mirlin, and D. G. Polyakov, Physica E 40, 1332 (2008).

${ }^{50}$ A. Chang and D. C. Tsui, Solid State Commun. 56, 153 (1985).

${ }^{51}$ T. Rotger, G. J. C. L. Bruls, J. C. Maan, P. Wyder, K. Ploog, and G. Weimann, Phys. Rev. Lett. 62, 90 (1989).

${ }^{52}$ H. P. Wei, D. C. Tsui, M. A. Paalanen, and A. M. M. Pruisken, Phys. Rev. Lett. 61, 1294 (1988).

${ }^{53}$ S. H. Simon and B. I. Halperin, Phys. Rev. Lett. 73, 3278 (1994).

${ }^{54}$ T. Ando, J. Phys. Soc. Jpn. 37, 1233 (1974).

${ }^{55}$ T. Ando, Y. Matsumoto, and Y. Uemura, J. Phys. Soc. Jpn. 39, 279 (1975).

${ }^{56}$ A. Isihara and L. Smrcka, J. Phys. C 19, 6777 (1986).

${ }^{57}$ S. Das Sarma and F. Stern, Phys. Rev. B 32, 8442 (1985).

${ }^{58}$ V. Umansky, R. de-Picciotto, and M. Heiblum, Appl. Phys. Lett. 71, 683 (1997).

${ }^{59}$ R. G. Mani et al. (unpublished). 